\title{
FREE AND PROPERLY DISCONTINUOUS ACTIONS OF GROUPS ON HOMOTOPY $2 n$-SPHERES
}

\author{
MAREK GOLASIŃSKI, DACIBERG LIMA GONÇALVES AND ROLANDO JIMÉNEZ
}

\begin{abstract}
Let $G$ be a group acting freely, properly discontinuously and cellularly on a finite dimensional $C \mathrm{~W}$-complex $\Sigma(2 n)$ which has the homotopy type of the $2 n$ - sphere $\mathbb{S}^{2 n}$. Then, this action induces an action of the group $G$ on the top cohomology of $\Sigma(2 n)$. For the family of virtually cyclic groups, we classify all groups which act on $\Sigma(2 n)$, the homotopy type of all possible orbit spaces and all actions on the top cohomology as well.

Under the hypothesis that $\operatorname{dim} \Sigma(2 n) \leq 2 n+1$, we study the groups with the virtual cohomological dimension $\operatorname{vcd} G<\infty$ which act as above on $\Sigma(2 n)$. It turns out that they consist of free groups and certain semi-direct products $F \rtimes \mathbb{Z}_{2}$ with $F$ a free group. For those groups $G$ and a given action of $G$ on $\operatorname{Aut}(\mathbb{Z})$, we present an algebraic criterion equivalent to the realizability of an action $G$ on $\Sigma(2 n)$ which induces the given action on its top cohomology. Then, we obtain a classification of those groups together with actions on the top cohomology of $\Sigma(2 n)$.
\end{abstract}

Introduction. The statement of the spherical space form problem in dimension $n$ is: classify all manifolds with the $n$-sphere $\mathbb{S}^{n}$ as the universal cover. Consequently, manifolds with finite fundamental groups. The development of that motivates classifications of the possible groups (not necessarily finite) which act freely, properly discontinuously and cellularly on an $n$-homotopy sphere $\Sigma(n)$ (a finite dimension $C W$-complex with the homotopy type of the $n$-sphere $\left.\mathbb{S}^{n}\right)$. Further, this

2010 Mathematics Subject Classification: primary: 57S30; secondary: 20F50, 20J06, 57Q91.

Key words and phrases: homotopy sphere, orbit space, cohomological (virtual) dimension, proper discontinuous and cellular action, virtually cyclic group.

The first and second authors gratefully acknowledge a support by Instituto de Matemáticas, UNAM, Oaxaca Branch, where the main part of this paper has been discussed. The authors were partially supported by CONACyT Grant 98697 and the third author was also supported by CONACyT Grant 151338. 
development began to accelerate with the discovery by J. Milnor [15] that some periodic groups could not act freely on any sphere. Then, R. Swan [25] showed that every periodic finite group acts freely on a finite $C W$-complex homotopic to $\mathbb{S}^{k m-1}$ for some $k$, where $m$ is the period of the group. Finite groups with such actions on $\Sigma(n)$ have been fully classified by Suzuki-Zassenhasus, see e.g. [1, Chapter IV, Theorem 6.15].

A free action of a discrete (finite or infinite) group $G$ on $\Sigma(n)$ induces an action on $H^{n}(\Sigma(n), \mathbb{Z})$, i.e., a homomorphism $G \rightarrow \operatorname{Aut}\left(H^{n}(\Sigma(n), \mathbb{Z})\right)$. Following [4, Proposition 10.2], for any action of a finite group $G$ on $\Sigma(2 n+1)$, the induced action $G \rightarrow \operatorname{Aut}\left(H^{2 n+1}(\Sigma(2 n+1), \mathbb{Z})\right)$ is trivial. On the other hand, in view of [26], the only finite groups acting freely on $\Sigma(2 n)$ are, up to isomorphism, trivial or $\mathbb{Z}_{2}$ and the induced homomorphism $\mathbb{Z}_{2} \rightarrow \operatorname{Aut}\left(H^{2 n}(\Sigma(2 n), \mathbb{Z})\right)$ is non-trivial. If the group $G$ is infinite there are more possibilities for the induced action of $G$ on $H^{n}(\Sigma(n), \mathbb{Z})$ than in the finite case, and that is a part of the problem to characterize those induced actions.

Actions of infinite discrete groups on $\Sigma(n)$ have been also studied, where the induced action on its top cohomology is in general non-trivial and it is an important part of the data. We state below some of the relevant results about this study motivated by a problem raised by C.T.C. Wall [32, p. 518]: whether any countable group with periodic Farell cohomology can act freely and properly on some product $\mathbb{R}^{m} \times \mathbb{S}^{n}$ ?

A breakthrough on Wall's question was made by Connolly and Prassidis (1989). In view of [6, Corollary 1.4], a discrete group $G$ with the virtual cohomological dimension $\operatorname{vcd} G<\infty$ acts freely and properly on $\mathbb{R}^{m} \times \mathbb{S}^{n}$ for some $m, n$ if and only if $G$ is countable and the Farrell cohomology $\hat{H}^{*}(G,-)$ is periodic (see [6] for the definition of the periodicity). Using arguments presented in [6], it was proved in [14, Proposition 9.1 and Proposition 9.3] that if a group $G$ which is not torsion-free with vcd $G<\infty$ acts freely and properly discontinuously on $\mathbb{R}^{m} \times \mathbb{S}^{n}$ then the period of $\hat{H}^{*}(G, \mathbb{Z})$ is two or divides $n+1$ provided either $n$ is even or odd, respectively.

It follows from [2] that a discrete group $G$ has periodic cohomology (after $d$-steps with $d \geq 0)$ if there is a positive integer $q$ and a class $\alpha \in H^{q}(G, \mathbb{Z})$ such that the cup product map

$$
\alpha \cup-: H^{i}(G, M) \longrightarrow H^{i+q}(G, M)
$$

is an isomorphism for every $G$-module $M$ and $i>d$. The result [2, Corollary 1.3] characterizes groups which act freely and properly discontinuously on $\mathbb{R}^{m} \times \mathbb{S}^{n}$, 
extends Wall's question above for groups with finite virtual cohomological dimension and states: $A$ discrete group $G$ acts freely and properly on $\mathbb{R}^{m} \times \mathbb{S}^{n}$ for some $m, n>0$ if and only if $G$ is a countable group with periodic cohomology.

Further, the result of Johnson [13, Theorem on p. 387] states:

Let $G$ be a group. Then the following are equivalent:

(i) there is a manifold $M$ of type $K(G, 1)$;

(ii) there is a covering action of $G$ on $\mathbb{R}^{m}$ for some $m$;

(iii) $G$ is countable and has finite cohomological dimension.

Consequently, such a group $G$ acts freely and properly discontinuously on $\mathbb{R}^{m} \times \mathbb{S}^{n}$ for any $n>0$. For more about this subject, we refer the reader to the papers [2], [6], [16], [20] and [31].

By O. Talelli [28], a group $G$ is said to have periodic cohomology (after $d$-steps with $d \geq 0)$ if there is a positive integer $q$ such that the functors $H^{i}(G,-)$ and $H^{i+q}(G,-)$ are naturally equivalent for $i>d$. The class of finite periodic groups has been extended (see e.g., [16] and [29]) to a larger class of discrete groups for which natural equivalences $H^{i}(G,-) \cong H^{i+q}(G,-)$ of functors for $i>d$ are given by cup product. We point out that it is an open problem if that periodicity is always induced by cup product with a cohomology class (see e.g., [2, Remark 2.12]).

It is not clear how to apply most of the results and techniques which appear in [2] and [6] to the cases $n=1$ and $n$ even. The study of properly discontinuous and cellular actions of discrete groups on a homotopy circle $\Sigma(1)$ was done in [10] using different methods than those in the papers mentioned above.

The purpose of this paper is to study free, properly discontinuous and cellular actions of infinite groups $G$ on $\Sigma(2 n)$. This study takes also into account the induced actions of $G$ on $H^{2 n}(\Sigma(2 n), \mathbb{Z})$.

In virtue of [14, Proposition 7.1]: the group $G$ is torsion-free or $G \cong G_{0} \rtimes \mathbb{Z}_{2}$ with a torsion-free subgroup $G_{0}$ provided $G$ acts freely and properly discontinuously on $\mathbb{R}^{m} \times \mathbb{S}^{2 n}$.

For actions of virtually cyclic groups we show:

Proposition 2.3. Let $G \times \Sigma(2 n) \rightarrow \Sigma(2 n)$ be an action of a non-trivial virtually cyclic group $G$ on $\Sigma(2 n)$ and $\varphi: G \rightarrow \operatorname{Aut}\left(H^{2 n}(\Sigma(2 n), \mathbb{Z})\right)$ the induced homomorphism. Then:

(1) $G$ is isomorphic to one of the groups: $\mathbb{Z}_{2}, \mathbb{Z}, \mathbb{Z} \oplus \mathbb{Z}_{2}$ or $\mathbb{Z}_{2} * \mathbb{Z}_{2} \cong \mathbb{Z} \rtimes \mathbb{Z}_{2}$;

(2) any of the groups above admits an action on some $\Sigma(2 n)$ and the pair $(G, \varphi)$ is realizable provided: 
(i) $G \cong \mathbb{Z}_{2}$ and $\varphi$ is non-trivial;

(ii) $G \cong \mathbb{Z}$ and $\varphi$ is any homomorphism;

(iii) $G \cong \mathbb{Z} \oplus \mathbb{Z}_{2}$, the restriction $\left.\varphi\right|_{\mathbb{Z}}$ is trivial and $\left.\varphi\right|_{\mathbb{Z}_{2}}$ is non-trivial;

(iv) $G \cong \mathbb{Z} \rtimes \mathbb{Z}_{2}$, the restriction $\left.\varphi\right|_{\mathbb{Z}}$ is trivial and $\left.\varphi\right|_{\mathbb{Z}_{2}}$ is non-trivial.

Further, the orbit space $\Sigma(2 n) / G$ has the homotopy type of one of the manifolds: $\mathbb{R} P^{2 n}, \mathbb{S}^{2 n} \times \mathbb{S}^{1}, \mathbb{S}^{2 n} \tilde{\times} \mathbb{S}^{1}$ (the only non-trivial $\mathbb{S}^{2 n}$-bundle over $\left.\mathbb{S}^{1}\right), \mathbb{R} P^{2 n} \times \mathbb{S}^{1}$ or $\mathbb{R} P^{2 n+1} \sharp \mathbb{R} P^{2 n+1}$.

Let $F$ be a free group. Given homomorphisms $\theta: \mathbb{Z}_{2} \rightarrow \operatorname{Aut}(F)$ and $\varphi: F \rtimes_{\theta} \mathbb{Z}_{2} \rightarrow$ Aut $(\mathbb{Z}) \cong \mathbb{Z}_{2}$ with $\left.\varphi\right|_{\mathbb{Z}_{2}}=\operatorname{id}_{\mathbb{Z}_{2}}$, we say that the pair $(\theta, \varphi)$ is realizable if there is an action

$$
\left(F \rtimes_{\theta} \mathbb{Z}_{2}\right) \times \Sigma(2 n) \rightarrow \Sigma(2 n)
$$

such that the induced homomorphism $F \rtimes_{\theta} \mathbb{Z}_{2} \rightarrow \operatorname{Aut}\left(H^{2 n}(\Sigma(2 n), \mathbb{Z})\right)$ coincides with $\varphi: F \rtimes_{\theta} \mathbb{Z}_{2} \rightarrow$ Aut $(\mathbb{Z})$. The key Lemma 3.1 states the necessary and sufficient conditions for a pair $(\theta, \varphi)$ to be realizable.

For a free group $F_{m}$ of finite rank $m \geq 1$, we define $m \times m$-matrices $A(k, r, s)$ over the integers which satisfy $A(k, r, s)^{2}=I_{m}$ with $k$ matrices $\left(\begin{array}{ll}0 & 1 \\ 1 & 0\end{array}\right)$, the identity matrix $I_{r}$ and $-I_{s}$ on the diagonal for $m=2 k+r+s$. Then, we make use of the the well-known representation $\rho_{m}$ : Aut $\left(F_{m}\right) \rightarrow G L_{m}(\mathbb{Z})$ to prove the following:

Theorem 3.4. Let $F_{m}=\left\langle x_{1}, \ldots, x_{m}\right\rangle$ be a free group with $m \geq 1, \theta: \mathbb{Z}_{2} \rightarrow$ $\operatorname{Aut}\left(F_{m}\right)$ and $\varphi: F_{m} \rtimes_{\theta} \mathbb{Z}_{2} \rightarrow \operatorname{Aut}(\mathbb{Z})$ be homomorphisms such that $\rho_{m}\left(\theta\left(1_{2}\right)\right)=$ $A(k, r, s)$ and $\left.\varphi\right|_{\mathbb{Z}_{2}}=\mathrm{id}_{\mathbb{Z}_{2}}$. Then the pair $(\theta, \varphi)$ is realizable if and only if $\varphi\left(x_{l}, 0\right)=0$ for $l=2 k+r+1, \ldots, 2 k+r+s$.

The result [8, Theorem 3] shows that given a free group $F_{m}$ and $\theta$ it is always possible to find a basis $\left\{x_{1}, \ldots, x_{m}\right\}$ for $F_{m}$ which satisfies the hypothesis of the theorem above.

This paper is organized into four sections, besides this Introduction.

In Section 1 basic facts on actions of groups on $\Sigma(2 n)$ are presented.

Section 2 aims to determine all non-trivial virtually cyclic groups admitting such actions on $\Sigma(2 n)$ and homotopy types of orbit spaces. The main result is Proposition 2.3. but Corollary 2.3 classifies orbit spaces of free and properly discontinuous actions of finite groups on certain spaces having universal covering $\mathbb{R} \times \mathbb{S}^{2 n}$.

Section 3 analyzes actions $G \times \Sigma(2 n) \rightarrow \Sigma(2 n)$ with the virtual cohomological dimension vcd $G<\infty$ and $\operatorname{dim} \Sigma(2 n) \leq 2 n+1$. We prove Theorem 3.4 and make 
some comments in Remark 3.5 about a version of this theorem for $F$ a free group of infinite rank.

Finally, in Section 4 we pose a question about actions of infinite discrete groups on $\mathbb{R}^{m} \times \mathbb{S}^{2 n}$.

Acknowledgments. The authors are indebted to F.X. Connolly and S. Prasidis for fruitful discussions on many aspects of this paper, in particular, on the current status of the Wall problem [32, p. 518] summarized in Section 4.

1. Preliminaries. A $C W$-complex $\Sigma(n)$ is said to be an n-homotopy sphere, if $\operatorname{dim} \Sigma(n)<\infty$ and there is a homotopy equivalence $\Sigma(n) \simeq \mathbb{S}^{n}$ for the $n$-sphere $\mathbb{S}^{n}$ with $n \geq 1$.

From now on, we assume that any action $G \times \Sigma(n) \rightarrow \Sigma(n)$ of a group $G$ on $\Sigma(2 n)$ is free, properly discontinuous and cellular. In the beginning of [10, Section 1], we have stated:

Remark 1.1. Notice that $n \leq \operatorname{dim} \Sigma(n)$ and for an action $G \times \Sigma(n) \rightarrow \Sigma(n)$ there is a fibration

$$
\Sigma(n) \rightarrow \Sigma(n) / G \rightarrow K(G, 1) .
$$

Consequently, there are isomorphisms $\pi_{k}(\Sigma(n)) \cong \pi_{k}(\Sigma(n) / G)$ for $k>1$ and $n \geq 1$, $\pi_{1}(\Sigma(n) / G) \cong G$ for $n>1$ and there is an extension

$$
e \rightarrow \mathbb{Z} \rightarrow \pi_{1}(\Sigma(1) / G) \rightarrow G \rightarrow e
$$

of groups.

Write cd $G$ (resp. ved $G$ ) for cohomological (resp. virtual cohomological) dimension of a group $G$ [4, Chapter VIII].

Given an action $G \times \Sigma(2 n) \rightarrow \Sigma(2 n)$, we consider the induced homomorphism

$$
G \rightarrow \operatorname{Aut}\left(H^{2 n}(\Sigma(2 n), \mathbb{Z})\right) \cong \mathbb{Z}_{2}
$$

which we call from now on the orientation of the $G$-action.

Then, we make use of [14, Proposition 7.1] and [26] to show:

Proposition 1.2. Let $G \times \Sigma(2 n) \rightarrow \Sigma(2 n)$ be an action of a group $G$ on $\Sigma(2 n)$. Then:

(1) $G \cong \mathbb{Z}_{2}$ or $G=E$ provided $G$ is finite. Further, $\mathbb{Z}_{2} \rightarrow \operatorname{Aut}\left(H^{2 n}(\Sigma(2 n), \mathbb{Z})\right)$ is non-trivial; 
(2) $G$ is torsion-free or $G \cong G_{0} \rtimes \mathbb{Z}_{2}$ for some torsion-free subgroup group $G_{0}$ of G.

Proof. (1) If $G$ is finite, then by [26], $G \cong \mathbb{Z}_{2}$ or $G=E$. Suppose that $\mathbb{Z}_{2} \rightarrow \operatorname{Aut}\left(H^{2 n}(\Sigma(2 n), \mathbb{Z})\right)$ is trivial. Then the Leray-Serre spectral sequence $E_{2}^{p, q}=$ $H^{p}\left(\mathbb{Z}_{2}, H^{q}(\Sigma(2 n), \mathbb{Z})\right)$ determined by the fibration

$$
\Sigma(2 n) \rightarrow \Sigma(2 n) / \mathbb{Z}_{2} \rightarrow K\left(\mathbb{Z}_{2}, 1\right)
$$

collapses. Hence, the group $H^{*}\left(\Sigma(2 n) / \mathbb{Z}_{2}, \mathbb{Z}\right)$ does not vanish for infinite many values of $*$, which contradicts the fact that $\operatorname{dim} \Sigma(2 n) / \mathbb{Z}_{2}<\infty$.

(2) Suppose $G$ is not torsion-free. Then, in view of (1), the induced action $G \rightarrow \operatorname{Aut}\left(H^{2 n}(\Sigma(2 n)), \mathbb{Z}\right)$ is onto and $G_{0}=\operatorname{ker}\left(G \rightarrow \operatorname{Aut}\left(H^{2 n}(\Sigma(2 n), \mathbb{Z})\right) \cong \mathbb{Z}_{2}\right)$ is torsion-free. Further, the extension

$$
e \rightarrow G_{0} \rightarrow G \rightarrow \mathbb{Z}_{2} \rightarrow e
$$

splits. Consequently, there is an isomorphism $G \cong G_{0} \rtimes \mathbb{Z}_{2}$.

Notice that from Proposition 1.2 it follows: if

$$
\varphi: G \cong G_{0} \rtimes \mathbb{Z}_{2} \rightarrow \operatorname{Aut}\left(H^{2 n}(\Sigma(2 n), \mathbb{Z})\right) \cong \mathbb{Z}_{2}
$$

is the induced action then the restriction $\left.\varphi\right|_{\mathbb{Z}_{2}}=\mathrm{id}_{\mathbb{Z}_{2}}$.

In [10, Proposition 1.7], we have shown:

Proposition 1.3. If ved $G<\infty$ and there is an action $G \times \Sigma(n) \rightarrow \Sigma(n)$ then $\operatorname{vcd} G \leq \operatorname{dim} \Sigma(n)-n$ for $n \geq 1$. In particular, $G$ is finite provided $\operatorname{dim} \Sigma(n)=n$.

Then, we deduce:

Corollary 1.4. If $G \times \Sigma(2 n) \rightarrow \Sigma(2 n)$ is an action with $\operatorname{dim} \Sigma(2 n) \leq 2 n+m$ and $\operatorname{vcd} G<\infty$ then $\operatorname{cd} G \leq m$ or $G \cong G_{0} \rtimes \mathbb{Z}_{2}$ with cd $G_{0} \leq m$. In particular, if $m=1$ then the group $G$ is free or $G \cong F \rtimes \mathbb{Z}_{2}$ for some free group $F$.

Proof. For an action $G \times \Sigma(2 n) \rightarrow \Sigma(2 n)$ with $\operatorname{dim} \Sigma(2 n) \leq 2 n+m$ and $\operatorname{ved} G<$ $\infty$, Proposition 1.3 yields vcd $G \leq m$. Then, Proposition 1.2 and [23] lead to $\operatorname{cd} G \leq$ $m$ or $G \cong G_{0} \rtimes \mathbb{Z}_{2}$ with cd $G_{0} \leq m$.

If $m=1$ then, by means of the above, [24] and [27], the group $G$ is free or $G \cong F \rtimes \mathbb{Z}_{2}$ for some free group $F$.

Now, we show that the family of groups $F \rtimes \mathbb{Z}_{2}$ for a free group $F$ is closed with respect to free products. 
Proposition 1.5. If $F_{i}$ are free groups for $i \in I$ then there is an isomorphism

$$
*_{i \in I}\left(F_{i} \rtimes \mathbb{Z}_{2}\right) \cong F \rtimes \mathbb{Z}_{2}
$$

for some free group $F$.

Proof. Given $\mathbb{Z}_{2}=\left\langle a_{i}\right\rangle$ for $i \in I$, write $\tilde{F}=\left\langle x_{i} \mid i \in I \backslash\left\{i_{0}\right\}\right\rangle$ for the free group generated by the set $\left\{x_{i} \mid i \in I \backslash\left\{i_{0}\right\}\right\}$ for a fixed $i_{0} \in I$. Further, consider the homomorphism $\theta: \mathbb{Z}_{2}=\langle b\rangle \rightarrow \operatorname{Aut}(\tilde{F})$ such that $\theta(b)\left(x_{i}\right)=x_{i}^{-1}$ for $i \in I \backslash\left\{i_{0}\right\}$. Then, the map

$$
\varphi: \tilde{F} \rtimes_{\theta} \mathbb{Z}_{2} \longrightarrow *_{i \in I} \mathbb{Z}_{2}
$$

given by $\varphi\left(x_{i}, 0\right)=a_{i} * a_{i_{0}}$ for $i \in I \backslash\left\{i_{0}\right\}$, and $\varphi(e, b)=a_{i_{0}}$ leads to an isomorphism

$\tilde{F} \rtimes_{\theta} \mathbb{Z}_{2} \stackrel{\cong}{\rightrightarrows} *_{i \in I} \mathbb{Z}_{2}$, so the group $\tilde{F}$ can be regarded as a subgroup of the main group via this isomorphism.

Next, consider the split epimorphism

$$
p: *_{i \in I}\left(F_{i} \rtimes \mathbb{Z}_{2}\right) \longrightarrow \mathbb{Z}_{2},
$$

where $\left.p\right|_{F_{i} \rtimes \mathbb{Z}_{2}}: F_{i} \rtimes \mathbb{Z}_{2} \rightarrow \mathbb{Z}_{2}$ is the projection map for all $i \in I$.

Notice that $\tilde{F} *\left(*_{i \in I}\left(F_{i} \times\{0\}\right)\right) \subseteq \operatorname{Ker} p$ and $\tilde{F} *\left(*_{i \in I}\left(F_{i} \times\{0\}\right)\right)$ is a normal subgroup of $\left(*_{i \in I}\left(F_{i} \rtimes \mathbb{Z}_{2}\right)\right.$. Further, $\left(g_{i_{1}}, 1\right) * \cdots *\left(g_{i_{n}}, 1\right) \in \operatorname{Ker} p$ yields that $n$ is even. This shows that $\operatorname{Ker} p=\tilde{F} *\left(*_{i \in I}\left(F_{i} \times\{0\}\right)\right.$ and the proof is complete.

2. Virtually cyclic groups acting on $\Sigma(2 n)$. Recall that a virtually cyclic group is a group that has a cyclic subgroup of finite index. The following criterion is mainly due to P. Scott and C.T.C. Wall [22]:

Theorem 2.1. Let $G$ be a finitely generated group. Then, the following are equivalent:

(1) $G$ is a group with two ends;

(2) $G$ has an infinite cyclic group of finite index;

(3) $G$ has a finite normal subgroup $F \unlhd G$ with the quotient $G / F \cong \mathbb{Z}$ or $\mathbb{Z}_{2} \star \mathbb{Z}_{2} \cong D_{\infty}$, the infinite dihedral group.

Equivalently, $G$ is of the form:

(1) a semi-direct product $F \rtimes \mathbb{Z}$ with $F$ finite or

(2) $G_{1} \star_{F} G_{2}$ with $F$ finite, where $\left[G_{i}: F\right]=2$ for $i=1,2$. 
Given an action $G \times \Sigma(n) \rightarrow \Sigma(n)$, write $\alpha_{\Sigma(n)}$ for the first Postnikov invariant [21] of the orbit space $\Sigma(n) / G$. In the sequel we need:

Lemma 2.2. Let a discrete group $G$ act on $\Sigma_{1}(n)$ and $\Sigma_{2}(n)$ with $\operatorname{dim} \Sigma_{1}(n) / G \leq$ $n+1$ for $n \geq 2$, and $\operatorname{dim} \Sigma_{2}(n) / G$ arbitrary.

The orbit spaces $\Sigma_{1}(n) / G$ and $\Sigma_{2}(n) / G$ have the same homotopy type if and only if there is an automorphism $\varphi \in \operatorname{Aut}(G)$ with $\varphi^{*}\left(\alpha_{\Sigma_{2}(n)}\right)=\alpha_{\Sigma_{1}(n)}$.

Proof. If the orbit spaces $\Sigma_{1}(n) / G$ and $\Sigma_{2}(n) / G$ have the same homotopy type, then certainly there is $\varphi \in \operatorname{Aut}(G)$ with $\varphi^{*}\left(\alpha_{\Sigma_{2}(n)}\right)=\alpha_{\Sigma_{1}(n)}$.

Now, suppose that there is $\varphi \in \operatorname{Aut}(G)$ with $\varphi^{*}\left(\alpha_{\Sigma_{2}(n)}\right)=\alpha_{\Sigma_{1}(n)}$. Then we derive a map of two stage Postnikov towers of $\Sigma_{1}(n) / G$ and $\Sigma_{2}(n) / G$ :

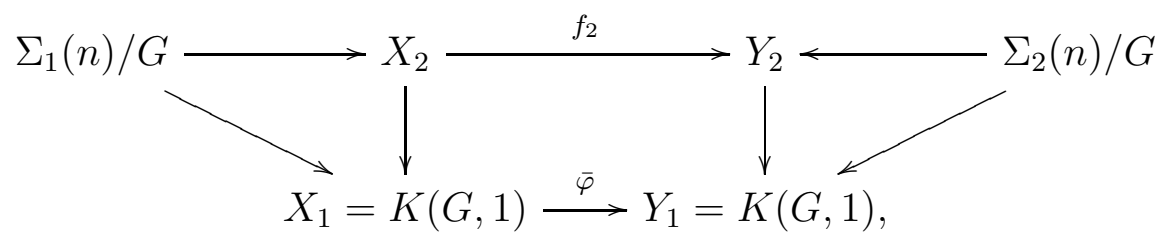

where $\bar{\varphi}$ is the induced map by $\varphi \in \operatorname{Aut}(G)$ at the Eilenberg-MacLane space $K(G, 1)$.

Because $\operatorname{dim} \Sigma_{1}(n) \leq n+1$, obstruction theory leads to a map $f: \Sigma_{1}(n) / G \rightarrow$ $\Sigma_{2}(n) / G$ with $\pi_{n}(f)=\pi_{n}\left(f_{2}\right): \pi_{n}\left(\Sigma_{1}(n) / G\right) \rightarrow \pi_{n}\left(\Sigma_{2}(n) / G\right)$ being an isomorphism. Then, for the lifting $\tilde{f}$

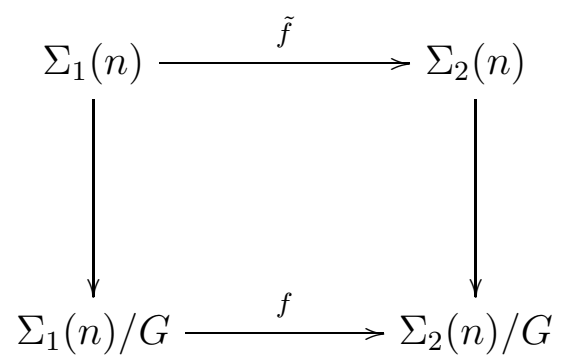

of $f: \Sigma_{1}(n) / G \rightarrow \Sigma_{2}(n) / G$, we deduce that $\pi_{n}(\tilde{f}): \pi_{n}\left(\Sigma_{1}(n)\right) \rightarrow \pi_{n}\left(\Sigma_{2}(n)\right)$ is an isomorphism. So we have an isomorphism in homology in all dimensions since the spaces have the homotopy type of a sphere. Therefore $\pi_{k}(\tilde{f})$ is an isomorphism for all $k$ and consequently the same for $\pi_{k}(f)$. Consequently, $f: \Sigma_{1}(n) / G \rightarrow \Sigma_{2}(n) / G$ is a homotopy equivalence, which completes the proof.

Let $G$ be a group and $\varphi: G \rightarrow \operatorname{Aut}(\mathbb{Z})$ a homomorphism. We say that the pair $(G, \varphi)$ is realizable if there is an action $G \times \Sigma(2 n) \rightarrow \Sigma(2 n)$ that the induced homomorphism $G \rightarrow \operatorname{Aut}\left(H^{2 n}(\Sigma(2 n), \mathbb{Z})\right)$ coincides with $\varphi: G \rightarrow \operatorname{Aut}(\mathbb{Z})$.

Then, we are in position to show: 
Proposition 2.3. Let $G \times \Sigma(2 n) \rightarrow \Sigma(2 n)$ be an action of a non-trivial virtually cyclic group $G$ on $\Sigma(2 n)$ and $\varphi: G \rightarrow \operatorname{Aut}\left(H^{2 n}(\Sigma(2 n), \mathbb{Z})\right)$ the induced homomorphism. Then:

(1) $G$ is isomorphic to one of the groups: $\mathbb{Z}_{2}, \mathbb{Z}, \mathbb{Z} \oplus \mathbb{Z}_{2}$ or $\mathbb{Z}_{2} * \mathbb{Z}_{2} \cong \mathbb{Z} \rtimes \mathbb{Z}_{2}$;

(2) any of the groups above admits an action on some $\Sigma(2 n)$ and the pair $(G, \varphi)$ is realizable provided:

(i) $G \cong \mathbb{Z}_{2}$ and $\varphi$ is non-trivial;

(ii) $G \cong \mathbb{Z}$ and $\varphi$ is any homomorphism;

(iii) $G \cong \mathbb{Z} \oplus \mathbb{Z}_{2}$, the restriction $\left.\varphi\right|_{\mathbb{Z}}$ is trivial and $\left.\varphi\right|_{\mathbb{Z}_{2}}$ is non-trivial;

(iv) $G \cong \mathbb{Z} \rtimes \mathbb{Z}_{2}$, the restriction $\left.\varphi\right|_{\mathbb{Z}}$ is trivial and $\left.\varphi\right|_{\mathbb{Z}_{2}}$ is non-trivial.

Further, the orbit space $\Sigma(2 n) / G$ has the homotopy type of one of the manifolds: $\mathbb{R} P^{2 n}, \mathbb{S}^{1} \times \mathbb{S}^{2 n}, \mathbb{S}^{1} \tilde{\times} \mathbb{S}^{2 n}$ (the only non-trivial $\mathbb{S}^{2 n}$-bundle over $\mathbb{S}^{1}$ being the mapping torus of the antipodal map $\left.\alpha: \mathbb{S}^{2 n} \rightarrow \mathbb{S}^{2 n}\right), \mathbb{S}^{1} \times \mathbb{R} P^{2 n}$ or $\mathbb{R} P^{2 n+1} \sharp \mathbb{R} P^{2 n+1}$.

Proof. (1): Follows immediately from Proposition 1.2 and Theorem 2.1 .

(2): Writing $\tilde{\mathbb{Z}}$ for the $G$-module structure on $H^{2 n}(\Sigma(2 n), \mathbb{Z}) \cong \mathbb{Z}$ for $G$ being one of the groups from (1), we make use of Lemma 2.2.

(i): $G \cong \mathbb{Z}_{2}$. Then, certainly there is the standard action $\mathbb{Z}_{2} \times \mathbb{S}^{2 n} \rightarrow \mathbb{S}^{2 n}$ for any $n \geq 1$ and by e.g., [3, Lemma 2.5], it holds that $\Sigma(2 n) / \mathbb{Z}_{2} \simeq \mathbb{R} P^{2 n}$, for any action $\mathbb{Z}_{2} \times \Sigma(2 n) \rightarrow \Sigma(2 n)$.

(ii): $G \cong \mathbb{Z}$. Because $H^{2 n+1}(\mathbb{Z}, \tilde{\mathbb{Z}})=H^{2 n+1}(\mathbb{Z}, \mathbb{Z})=0$, there is at most one homotopy type of $\Sigma(2 n) / \mathbb{Z}$ for the non-trivial and trivial $G$-actions on $H^{2 n}(\Sigma(2 n), \mathbb{Z})$. Any of them may be realized. Namely, consider the $\mathbb{Z}$-actions:

$$
\circ, \bar{\circ}: \mathbb{Z} \times\left(\mathbb{R} \times \mathbb{S}^{2 n}\right) \rightarrow \mathbb{R} \times \mathbb{S}^{2 n}
$$

given by $n \circ(t, x)=(t+n, x)$ and $n \bar{\circ}(t, x)=(t+n,-x)$, respectively for $n \in \mathbb{Z}$ and $(t, x) \in \mathbb{R} \times \mathbb{S}^{2 n}$. The corresponding orbit spaces are homotopic to $\mathbb{S}^{1} \times \mathbb{S}^{2 n}$ or $\mathbb{S}^{1} \tilde{\times} \mathbb{S}^{2 n}$, respectively.

(iii): $G \cong \mathbb{Z} \oplus \mathbb{Z}_{2}$. Then, by Proposition 1.2 and its proof, $G$ acts non-trivially on $H^{2 n}(\Sigma(2 n), \mathbb{Z}) \cong \mathbb{Z}$. Hence, we have an epimorphism $\varphi: \mathbb{Z} \oplus \mathbb{Z}_{2} \rightarrow \mathbb{Z}_{2}$ such that $\varphi\left(0,1_{2}\right)=1_{2}$ and $\varphi(1,0)=0$ or $\varphi(1,0)=1_{2}$. Further, observe that there is an isomorphism $\mathbb{Z} \oplus \mathbb{Z}_{2} \cong \mathbb{Z} \oplus \mathbb{Z}_{2}$ given by: $(1,0) \mapsto\left(1,1_{2}\right)$ and $\left(0,1_{2}\right) \mapsto\left(0,1_{2}\right)$.

Analysing the Lyndon-Hochschild-Serre spectral sequence corresponding to the extension

$$
e \rightarrow \mathbb{Z} \rightarrow \underset{9}{\oplus \mathbb{Z}} \underset{\mathbb{Z}_{2}}{\rightarrow} \mathbb{Z}_{2} \rightarrow e
$$


we deduce that $H^{0}\left(\mathbb{Z} \oplus \mathbb{Z}_{2}, \tilde{\mathbb{Z}}\right)=0$ and $H^{k}\left(\mathbb{Z} \oplus \mathbb{Z}_{2}, \tilde{\mathbb{Z}}\right) \cong \mathbb{Z}_{2}$ for $k>0$. In particular, $H^{2 n+1}\left(\mathbb{Z} \oplus \mathbb{Z}_{2}, \tilde{\mathbb{Z}}\right) \cong \mathbb{Z}_{2}$ and there are two possible values for the first Postnikov invariant $K\left(\mathbb{Z} \oplus \mathbb{Z}_{2}, 1\right) \rightarrow \widehat{K}(\mathbb{Z}, 2 n+1)$ of the orbit space $\Sigma(2 n) / \mathbb{Z} \oplus \mathbb{Z}_{2}$, where $\widehat{K}(\mathbb{Z}, 2 n+1)=K\left(\widetilde{\mathbb{Z} \oplus \mathbb{Z}_{2}}, 1\right) \times_{\mathbb{Z} \oplus \mathbb{Z}_{2}} K(\mathbb{Z}, 2 n+1)$ is the twisted EilenbergMacLane space. But, the Leray-Serre spectral sequence corresponding to the fibration $K(\mathbb{Z}, 2 n+1) \rightarrow X_{2} \rightarrow K\left(\mathbb{Z} \oplus \mathbb{Z}_{2}, 1\right)$ shows that this invariant cannot be trivial. Consequently, there is only one homotopy type of the quotient space realized by the action:

$$
\circ:\left(\mathbb{Z} \oplus \mathbb{Z}_{2}\right) \times\left(\mathbb{R} \times \mathbb{S}^{2 n}\right) \rightarrow \mathbb{R} \times \mathbb{S}^{2 n}
$$

given by $(1,0) \circ(t, x)=(t+1, x)$ and $\left(0,1_{2}\right) \circ(t, x)=(t,-x)$ for $(t, x) \in \mathbb{R} \times \mathbb{S}^{2 n}$ with the corresponding quotient space homotopic to $\mathbb{S}^{1} \times \mathbb{R} P^{2 n}$.

(iv): $G \cong \mathbb{Z} \rtimes \mathbb{Z}_{2}$. Then, again by [14, Proposition 7.1] and its proof, $G$ acts non-trivially on $H^{2 n}(\Sigma(2 n), \mathbb{Z}) \cong \mathbb{Z}$. Hence, we obtain an extension

$$
0 \rightarrow \mathbb{Z} \rightarrow \mathbb{Z} \rtimes \mathbb{Z}_{2} \rightarrow \mathbb{Z}_{2} \rightarrow 0 .
$$

Then, the corresponding Lyndon-Hochschild-Serre spectral sequence yields $H^{2 n+1}(\mathbb{Z} \rtimes$ $\left.\mathbb{Z}_{2}, \tilde{\mathbb{Z}}\right) \cong \mathbb{Z}_{2}$. The methods parallel to those in (iii) show that the first Postnikov invariant of the orbit space $\Sigma(2 n) / \mathbb{Z} \rtimes \mathbb{Z}_{2}$ cannot be trivial. Finally, there is also only one homotopy type of the quotient space realized by the action:

$$
\circ:\left(\mathbb{Z} \rtimes \mathbb{Z}_{2}\right) \times\left(\mathbb{R} \times \mathbb{S}^{2 n}\right) \rightarrow \mathbb{R} \times \mathbb{S}^{2 n}
$$

given by $(1,0) \circ(t, x)=(t+1, x)$ and $\left(0,1_{2}\right) \circ(t, x)=(-t,-x)$ for $(t, x) \in \mathbb{R} \times \mathbb{S}^{2 n}$ with the corresponding orbit space homotopic to $\mathbb{R} P^{2 n+1} \sharp \mathbb{R} P^{2 n+1}$.

The last statement follows from the proof of (2).

In view of [30, Corollary 2], the classification of all free and properly discontinuous actions by a finite group on $\mathbb{S}^{1} \times \mathbb{S}^{2}$ follows from the observation that there exist only four compact 3-manifolds which have $\mathbb{R} \times \mathbb{S}^{2}$ as a universal covering space.

Now, we deduce below that any manifold with the universal covering space $\mathbb{R} \times \mathbb{S}^{2 n}$ has the homotopy type one of the following manifolds: $\mathbb{S}^{1} \times \mathbb{S}^{2 n}, \mathbb{S}^{1} \tilde{\times} \mathbb{S}^{2 n}, \mathbb{S}^{1} \times \mathbb{R} P^{2 n}$ or $\mathbb{R} P^{2 n+1} \sharp \mathbb{R} P^{2 n+1}$.

Corollary 2.4. Suppose that a finite non-trivial group $G$ acts freely on one of the manifolds: $\mathbb{S}^{1} \times \mathbb{S}^{2 n}, \mathbb{S}^{1} \tilde{\times} \mathbb{S}^{2 n}, \mathbb{S}^{1} \times \mathbb{R} P^{2 n}$ or $\mathbb{R} P^{2 n+1} \sharp \mathbb{R} P^{2 n+1}$. Let $M$ be the orbit space of $G$.

(1) If $G$ acts on $\mathbb{S}^{1} \times \mathbb{S}^{2 n}$ then: 
(i) $G \cong \mathbb{Z}_{2}$ and $M \simeq \mathbb{S}^{1} \times \mathbb{S}^{2 n}, M \simeq \mathbb{S}^{1} \tilde{\times} \mathbb{S}^{2 n}, M \simeq \mathbb{S}^{1} \times \mathbb{R} P^{2 n}$ or

$M \simeq \mathbb{R} P^{2 n+1} \sharp \mathbb{R} P^{2 n+1}$;

(ii) $G \cong \mathbb{Z}_{2} \oplus \mathbb{Z}_{2}$ and $M \simeq \mathbb{S}^{1} \times \mathbb{R} P^{2 n}$ or $M \simeq \mathbb{R} P^{2 n+1} \sharp \mathbb{R} P^{2 n+1}$;

(iii) $G \cong \mathbb{Z}_{2 k+1}$ for some $k \geq 1$ and $M \simeq \mathbb{S}^{1} \times \mathbb{S}^{2 n}$;

(iv) $G \cong \mathbb{Z}_{2 k}$ for some $k>1$ and $M \simeq \mathbb{S}^{1} \times \mathbb{S}^{2 n}, M \simeq \mathbb{S}^{1} \tilde{x} \mathbb{S}^{2 n}$ or $M \simeq \mathbb{S}^{1} \times \mathbb{R} P^{2 n}$;

(v) $G \cong \mathbb{Z}_{2} \oplus \mathbb{Z}_{2 k}$ for some $k \geq 1$ and $M \simeq \mathbb{S}^{1} \times \mathbb{R} P^{2 n}$;

(vi) $G \cong \mathbb{Z}_{k} \rtimes \mathbb{Z}_{2}=D_{k}$ for some $k>2$, the dihedral group of order $2 k$ and $M \simeq \mathbb{R} P^{2 n+1} \sharp \mathbb{R} P^{2 n+1} ;$

(2) If $G$ acts on $\mathbb{S}^{1} \tilde{\times} \mathbb{S}^{2 n}$ then:

(i) $G \cong \mathbb{Z}_{2 k+1}$ for some $k \geq 1$ and $M \simeq \mathbb{S}^{1} \tilde{\times} \mathbb{S}^{2 n}$;

(ii) $G \cong \mathbb{Z}_{2 k}$ for some $k \geq 1$ and $M \simeq \mathbb{S}^{1} \times \mathbb{R} P^{2 n}$.

(3) If $G$ acts on $\mathbb{S}^{1} \times \mathbb{R} P^{2 n}$ then $G \cong \mathbb{Z}_{k}$ for some $k \geq 2$ and $M \simeq \mathbb{R} P^{2 n} \times \mathbb{S}^{1}$.

(4) If $G$ acts on $\mathbb{R} P^{2 n+1} \sharp \mathbb{R} P^{2 n+1}$ then $G \cong \mathbb{Z}_{2}$ and $M \simeq \mathbb{R} P^{2 n+1} \sharp \mathbb{R} P^{2 n+1}$.

Further, in all four cases above, the groups described act on the corresponding manifold.

Proof. We remark that $\mathbb{R} \times \mathbb{S}^{2 n}$ is the universal covering space of the manifolds listed above and make use of Proposition 2.3 .

(1): If $G$ acts on $\mathbb{S}^{1} \times \mathbb{S}^{2 n}$ then the quotient map $\mathbb{S}^{1} \times \mathbb{S}^{2 n} \rightarrow \mathbb{S}^{1} \times \mathbb{S}^{2 n} / G$ is covering and $\mathbb{S}^{1} \times \mathbb{S}^{2 n} / G$ is homotopic to one of those manifolds. Then, we get an extension of groups

$$
e \rightarrow \mathbb{Z} \rightarrow \pi \rightarrow G \rightarrow e
$$

where $\pi=\pi_{1}\left(\mathbb{S}^{1} \times / G \mathbb{S}^{2 n}\right)$. Certainly, $\mathbb{S}^{1} \times \mathbb{S}^{2 n} / G$ cannot be homeomorphic to $\mathbb{R} P^{n}$ because the group $G$ is finite.

If $\mathbb{S}^{1} \times \mathbb{S}^{2 n} / G \cong \mathbb{S}^{1} \times \mathbb{S}^{2 n}$ or $\mathbb{S}^{1} \times \mathbb{S}^{2 n} G \cong \mathbb{S}^{1} \tilde{\times} \mathbb{S}^{2 n}$ then $G \cong \mathbb{Z}_{k}$ for some $k \geq 2$.

If $\mathbb{S}^{1} \times \mathbb{S}^{2 n} / G \cong \mathbb{S}^{1} \times \mathbb{R} P^{n}$ then $G \cong \mathbb{Z}_{k}$ (for $k$ odd) or $G \cong \mathbb{Z}_{2} \oplus \mathbb{Z}_{k}$ (for $k$ even).

Because the manifold $\mathbb{S}^{1} \times \mathbb{S}^{2 n}$ is oriented and $\left(1_{2}, 2 k\right)$ is in the kernel of the epimorphism $\mathbb{Z}_{2} \times \mathbb{Z} \rightarrow \mathbb{Z}_{4 k} \rightarrow e$, the group $\mathbb{Z}_{4 k}$ cannot act freely on $\mathbb{S}^{1} \times \mathbb{S}^{2 n}$ to obtain $\mathbb{S}^{1} \times \mathbb{R} P^{2 n}$.

If $M \simeq \mathbb{R} P^{2 n+1} \sharp \mathbb{R} P^{2 n+1}$ then $G \cong \mathbb{Z}_{k} \rtimes \mathbb{Z}_{2}$, the dihedral group of order $2 k$ for some $k>2$.

(2): Because $\mathbb{S}^{1} \tilde{\times} \mathbb{S}^{2 n}$ is non-oriented, $\mathbb{S}^{1} \tilde{\times} \mathbb{S}^{2 n} / G \cong \mathbb{S}^{1} \tilde{\times} \mathbb{S}^{2 n}$ or $\mathbb{S}^{1} \tilde{\times} \mathbb{S}^{2 n} / G \cong \mathbb{S}^{1} \times$ $\mathbb{R} P^{2 n}$. Hence, $G \cong \mathbb{Z}_{k}$ for some $k>1$ or $G \cong \mathbb{Z}_{2} \oplus \mathbb{Z}_{k}$ for $k$ odd. But $\mathbb{S}^{1} \tilde{\times} \mathbb{S}^{2 n}$ is non-oriented, so for the epimorphism $\mathbb{Z}_{2} \oplus \mathbb{Z} \rightarrow \mathbb{Z}_{2} \oplus \mathbb{Z}_{k}$ some element $\left(1_{2}, l\right)$ with $l \neq 0$ must be in its kernel. Hence, $G \cong \mathbb{Z}_{k}$ for some $k>1$, only. 
(3): Because $\mathbb{S}^{1} \times \mathbb{R} P^{2 n}$ is non-oriented, $\mathbb{S}^{1} \times \mathbb{R} P^{2 n} / G \cong \mathbb{S}^{1} \tilde{\times} \mathbb{S}^{2 n}$ or $\mathbb{S}^{1} \times \mathbb{R} P^{2 n} / G \cong$ $\mathbb{S}^{1} \times \mathbb{R} P^{2 n}$. Then, we obtain that $G \cong \mathbb{Z}_{k}$ for some $k \geq 2$.

(4): Because $\pi_{1}\left(\mathbb{R} P^{2 n+1} \sharp \mathbb{R} P^{2 n+1}\right) \cong \mathbb{Z} \rtimes \mathbb{Z}_{2}$, we have only to analyze the extension

$$
e \rightarrow \mathbb{Z} \rtimes \mathbb{Z}_{2} \rightarrow \mathbb{Z} \rtimes \mathbb{Z}_{2} \rightarrow G \rightarrow e .
$$

Because $\mathbb{Z} \rtimes \mathbb{Z}_{2}$ must be sent to its normal subgroup by the monomorphism $e \rightarrow$ $\mathbb{Z} \rtimes \mathbb{Z}_{2} \rightarrow \mathbb{Z} \rtimes \mathbb{Z}_{2}$, we deduce that $G \cong \mathbb{Z}_{2}$.

Let $n \geq 2$ and let $\tau$ be a free involution on $\mathbb{S}^{1} \times \mathbb{S}^{n}$. Then, in view of [3, Theorem 2.1] the quotient $\mathbb{S}^{1} \times \mathbb{S}^{n} / \tau$ belongs to one of the four homotopy types: $\mathbb{S}^{1} \times \mathbb{S}^{n}$, $\mathbb{S}^{1} \tilde{\times} \mathbb{S}^{n}, \mathbb{S}^{1} \times \mathbb{R} P^{n}$ and $\mathbb{R} P^{n+1} \sharp \mathbb{R} P^{n+1}$ realized by the standard involutions.

Now, we are in position to conclude the following generalization of the above, provided $n$ is even:

Corollary 2.5. Let $n \geq 1$ and $\tau$ be a free involution on one the four manifolds: $\mathbb{S}^{1} \times \mathbb{S}^{2 n}, \mathbb{S}^{1} \tilde{\times} \mathbb{S}^{2 n}, \mathbb{S}^{1} \times \mathbb{R} P^{2 n}$ or $\mathbb{R} P^{2 n+1} \sharp \mathbb{R} P^{2 n+1}$. Then, the corresponding orbit space also belongs to one of their homotopy types.

3. Other groups acting on $\Sigma(2 n)$. Here, we analyze actions $G \times \Sigma(2 n) \rightarrow$ $\Sigma(2 n)$ with $\operatorname{vcd} G<\infty$ and $\operatorname{dim} \Sigma(2 n) \leq 2 n+1$. By Corollary 1.4, the group $G$ is free or $G \cong F \rtimes \mathbb{Z}_{2}$ for some free group $F$ with an arbitrary rank.

Let $F$ be a free group and Aut $(F)$ its automorphism group. For a homomorphism $\theta: \mathbb{Z}_{2} \rightarrow \operatorname{Aut}(F)$ we consider the semidirect product $G \cong F \rtimes_{\theta} \mathbb{Z}_{2}$ which is completely determined by $\theta$. Given also $\varphi: F \rtimes_{\theta} \mathbb{Z}_{2} \rightarrow$ Aut $(\mathbb{Z}) \cong \mathbb{Z}_{2}$ with $\left.\varphi\right|_{\mathbb{Z}_{2}}=\operatorname{id}_{\mathbb{Z}_{2}}$, we say that the pair $(\theta, \varphi)$ is realizable if $\left(F \rtimes_{\theta} \mathbb{Z}_{2}, \varphi\right)$ is realizable (see Section 1 ).

Notice that any free group $F$ acts on the homotopy $2 n$-sphere $\left(\widetilde{\bigvee_{i \in I} \mathbb{S}^{1}}\right) \times \mathbb{S}^{2 n}$ for any $n \geq 1$, where $\widetilde{\bigvee_{i \in I} \mathbb{S}^{1}}$ is the universal covering of the wedge $\bigvee_{i \in I} \mathbb{S}^{1}$ provided $F=\left\langle x_{i} ; i \in I\right\rangle$. Consequently, for the trivial homomorphism $\theta_{0}: \mathbb{Z}_{2} \rightarrow \operatorname{Aut}(F)$, any pair $\left(\theta_{0}, \varphi\right)$ is realizable by the action

$$
\left.\circ:\left(F \rtimes_{\theta} \mathbb{Z}_{2}\right) \times\left(\widetilde{\bigvee_{i \in I} \mathbb{S}^{1}}\right) \times \mathbb{S}^{2 n}\right) \rightarrow\left(\widetilde{\bigvee_{i \in I} \mathbb{S}^{1}}\right) \times \mathbb{S}^{2 n}
$$

given by: $(g, 0) \circ(t, s)=(g t, \operatorname{sgn}(g) s$,$) and \left(g, 1_{2}\right) \circ(t, s)=(g t,-\operatorname{sgn}(g) s)$ for $g \in F$ and $(t, s) \in\left(\widetilde{\bigvee_{i \in I} \mathbb{S}^{1}}\right) \times \mathbb{S}^{2 n}$, where sgn $: F \rightarrow \mathbb{Z}_{2}=\{ \pm 1\}$ is the homomorphism determined by the restriction of $\varphi: F \rtimes_{\theta} \mathbb{Z}_{2} \rightarrow$ Aut $(\mathbb{Z}) \cong \mathbb{Z}_{2}$ to the group $F$.

Writing $\mathbb{Z}_{2}=\left\langle 1_{2}\right\rangle$, we show a general fact: 
Lemma 3.1. (Fundamental Lemma) The pair $(\theta, \varphi)$ is realizable if and only if it does not exist $g \in F$ such that

$$
\left\{\begin{array}{l}
\theta\left(1_{2}\right)(g)=g^{-1} \\
\varphi(g, 0)=1_{2}
\end{array}\right.
$$

Proof. Let $\theta: \mathbb{Z}_{2} \rightarrow$ Aut $(F)$. By the 1-dimensional analog of the Nielsen realization problem [7, Theorems 2.1 and 4.1], the automorphism $\theta\left(1_{2}\right) \in \operatorname{Aut}(F)$ can be realized by a homeomorphism $h: \Gamma \rightarrow \Gamma$ of a graph $\Gamma$ with the fundamental group $\pi_{1}(\Gamma) \cong F$, such that $h$ has a fixed point and $h^{2}=\operatorname{id}_{\Gamma}$. Writing $\tilde{\Gamma}$ for the universal covering of $\Gamma$, we get the induced homeomorphism $\tilde{h}: \tilde{\Gamma} \rightarrow \tilde{\Gamma}$ with $\tilde{h}^{2}=\mathrm{id}_{\tilde{\Gamma}}$. Then, we are in a position to consider a map

$$
\circ:\left(F \rtimes_{\theta} \mathbb{Z}_{2}\right) \times\left(\tilde{\Gamma} \times \mathbb{S}^{2 n}\right) \rightarrow \tilde{\Gamma} \times \mathbb{S}^{2 n}
$$

given by: $(g, 0) \circ(t, s)=\left(\left(\theta\left(1_{2}\right) g\right) t, \operatorname{sgn}(g) s\right)$ and $\left(g, 1_{2}\right) \circ(t, s)=\left(\left(\theta\left(1_{2}\right) g\right)(\tilde{h}(t)),-\operatorname{sgn}(g) s\right)$ for $g \in F$ and $(t, s) \in \tilde{\Gamma} \times \mathbb{S}^{2 n}$. Now, we prove that the map defined above is an action of the group $F \rtimes_{\theta} \mathbb{Z}_{2}$. So for any two elements $w_{1}, w_{2} \in F \rtimes_{\theta} \mathbb{Z}_{2}$ and $(t, s) \in \tilde{\Gamma} \times \mathbb{S}^{2 n}$ we must show that $w_{2}\left(w_{1}(t, s)\right)=\left(w_{2} w_{1}\right)(t, s)$. Notice that $\operatorname{sgn}\left(g \theta\left(1_{2}\right) g^{\prime}\right)=\operatorname{sgn}\left(g g^{\prime}\right)$ and $\left(g, 1_{2}\right)(s, t)=\left((g, 0)\left(e, 1_{2}\right)\right)(t, s)=(g, 0)(\tilde{h}(t),-s)=\left(\left(\theta\left(1_{2}\right) g\right) \tilde{h}(t),-\operatorname{sgn}(g) s\right)$. The case where $w_{i}=\left(g_{i}, \overline{0}\right)$ for $i=1,2$ is easy and we leave to the reader. For the remaining cases we have:

(i) $\left((g, 0)\left(g^{\prime}, 1_{2}\right)\right)(t, s)=\left(g g^{\prime}, 1_{2}\right)(t, s)=\left(\left(\theta\left(1_{2}\right)\left(g g^{\prime}\right)\right) \tilde{h}(t),-\operatorname{sgn}\left(g g^{\prime}\right) s\right)$ and $\left.(g, 0)\left(\left(g^{\prime}, 1_{2}\right)\right)(t, s)\right)=(g, 0)=\left((g, 0)\left(g^{\prime}, 1_{2}\right)\right)(t, s)=(g, 0)\left(\left(\theta\left(1_{2}\right) g^{\prime}\right) \tilde{h}(t),-\operatorname{sgn}\left(g^{\prime}\right) s\right)=$ $\left.\left(\left(\theta\left(1_{2}\right) g\right)\left(\theta\left(1_{2}\right) g^{\prime}\right) \tilde{h}(t),-\operatorname{sgn}(g) \operatorname{sgn}\left(g^{\prime}\right) s\right)=(g, 0)\left(g^{\prime}, 1_{2}\right)\right)(t, s)$;

(ii) $\left(\left(g, 1_{2}\right)\left(g^{\prime}, 0\right)\right)(t, s)=\left(g \theta\left(1_{2}\right) g^{\prime}, 1_{2}\right)(t, s)=\left(\theta\left(1_{2}\right)\left(g \theta\left(1_{2}\right) g^{\prime}\right) \tilde{h}(t),-\operatorname{sgn}\left(g \theta\left(1_{2}\right) g^{\prime}\right) s\right)=$ $\left(\left(\theta\left(1_{2}\right) g\right) g^{\prime} \tilde{h}(t), \operatorname{sgn}\left(g g^{\prime}\right) s\right)$ and

$\left(g, 1_{2}\right)\left(\left(g^{\prime}, 0\right)(t, s)\right)=\left(g, 1_{2}\right)\left(\left(\theta\left(1_{2}\right) g^{\prime}\right) t, \operatorname{sgn}\left(g^{\prime}\right) s\right)=\left(\theta\left(1_{2}\right) g \tilde{h}\left(\theta\left(1_{2}\right) g^{\prime}\right)(t),-\operatorname{sgn}(g) \operatorname{sgn}\left(g^{\prime}\right) s\right)$ $=\left(\left(\theta\left(1_{2}\right) g\right) g^{\prime} \tilde{h}(t),-\operatorname{sgn}(g) \operatorname{sgn}\left(g^{\prime}\right) s\right)=\left(\left(g, 1_{2}\right)\left(g^{\prime}, 0\right)\right)(t, s)$;

(iii) $\left(\left(g, 1_{2}\right)\left(g^{\prime}, 1_{2}\right)\right)(t, s)=\left(g \theta\left(1_{2}\right) g^{\prime}, 0\right)(t, s)=\left(\left(\theta\left(1_{2}\right) g \theta\left(1_{2}\right) g^{\prime}\right) t,-\operatorname{sgn}\left(g \theta\left(1_{2}\right) g^{\prime}\right) s\right)=$ $\left.\left(\left(\theta\left(1_{2}\right) g\right) g^{\prime}\right) t,-\operatorname{sgn}\left(g g^{\prime}\right) s\right)$ and $\left(g, 1_{2}\right)\left(\left(g^{\prime}, 1_{2}\right)(t, s)\right)=\left(g, 1_{2}\right)\left(\left(\theta\left(1_{2}\right) g^{\prime}\right) \tilde{h}(t),-\operatorname{sgn}\left(g^{\prime}\right) s\right)=$ $\left.\left(\left(\theta\left(1_{2}\right) g\right) \tilde{h}\left(\left(\theta\left(1_{2}\right) g^{\prime}\right) \tilde{h}(t)\right),-\operatorname{sgn}(g) \operatorname{sgn}\left(g^{\prime}\right) s\right)=\left(\left(\theta\left(1_{2}\right) g\right) g^{\prime}\right) t,-\operatorname{sgn}(g) \operatorname{sgn}\left(g^{\prime}\right) s\right)=$ $\left(\left(g, 1_{2}\right)\left(g^{\prime}, 1_{2}\right)\right)(t, s)$.

Consequently, $\circ:\left(F \rtimes_{\theta} \mathbb{Z}_{2}\right) \times\left(\tilde{\Gamma} \times \mathbb{S}^{2 n}\right) \rightarrow \tilde{\Gamma} \times \mathbb{S}^{2 n}$ is a well-defined action. Because it does not exist $g \in F$ such that $\left\{\begin{array}{l}\theta\left(1_{2}\right)(g)=g^{-1}, \\ \varphi(g, 0)=1_{2}\end{array}\right.$ for any $g \in F$, the action $\circ$ : $\left(F_{m} \rtimes_{\theta} \mathbb{Z}_{2}\right) \times\left(\tilde{\Gamma} \times \mathbb{S}^{2 n}\right) \rightarrow \tilde{\Gamma} \times \mathbb{S}^{2 n}$ is free. Otherwise suppose that $\left(g, 1_{2}\right) \circ(t, s)=(t, s)$. 
Then we have $(t, s)=\left(\left(\theta\left(1_{2}\right) g\right)(\tilde{h}(t)),-\operatorname{sgn}(g) s\right)=\tilde{h}(g t)$ which implies $\operatorname{sgn}(g)=-1$ and $t=\left(\theta\left(1_{2}\right) g\right)(\tilde{h}(t))$. The second equation is equivalent to $\tilde{h}(t)=\tilde{h}^{2}(g t)=g t=$ $g \theta\left(1_{2}\right)(g) \tilde{h}(t)$ or $g \theta\left(1_{2}\right)(g)=1$. So the system of equations has a solution which is a contradiction. So we have a free, properly discontinuous and cellular action. Further, the induced homomorphism $\varphi: F \rtimes_{\theta} \mathbb{Z}_{2} \rightarrow \operatorname{Aut}\left(H^{2 n}\left(\tilde{\Gamma} \times \mathbb{S}^{2 n}\right), \mathbb{Z}\right)$ coincides with the given one $\varphi: F \rtimes_{\theta} \mathbb{Z}_{2} \rightarrow \operatorname{Aut}(\mathbb{Z})$.

Next, suppose that $\left\{\begin{array}{l}\theta\left(1_{2}\right)(g)=g^{-1}, \\ \varphi(g, 0)=1_{2}\end{array}\right.$ for some $g \in F$ and there is an action $\left(F \rtimes_{\theta}\right.$ $\left.\mathbb{Z}_{2}\right) \times \Sigma(2 n) \rightarrow \Sigma(2 n)$. Then, on one hand we have that $\varphi\left(g, 1_{2}\right)=\varphi(g, 0) \varphi\left(e, 1_{2}\right)=0$ and on the other hand, Proposition 1.2(1) leads to $\varphi\left(g, 1_{2}\right)=1_{2}$, because the order of $\left(g, 1_{2}\right) \in F \rtimes_{\theta} \mathbb{Z}_{2}$ is two. This contradiction completes the proof.

Corollary 3.2. The group $F \rtimes_{\theta} \mathbb{Z}_{2}$ acts on $\Sigma(2 n)=\tilde{\Gamma} \times \mathbb{S}^{2 n}$ for any $n \geq 1$, where $\Gamma$ is a graph (a finite graph provided $F$ is of finite rank) with $\pi_{1}(\Gamma)=F$.

Proof. Given the group $F \rtimes_{\theta} \mathbb{Z}_{2}$, consider the homomorphism $\varphi: F \rtimes_{\theta} \mathbb{Z}_{2} \rightarrow$ Aut $(\mathbb{Z})$ given by the projection map onto the second factor. Then, in view of Lemma 3.1. the pair $(\theta, \varphi)$ is realizable and this leads to an action of $F \rtimes_{\theta} \mathbb{Z}_{2}$ on $\Sigma(2 n)=$ $\tilde{\Gamma} \times \mathbb{S}^{2 n}$ for any $n \geq 1$, where $\Gamma$ is a graph (a finite one provided $F$ is of finite rank) with $\pi_{1}(\Gamma)=F$, and the result follows.

Now, let $F_{m}$ be the free group with finite rank $m \geq 1$. For $\theta: \mathbb{Z}_{2} \rightarrow \operatorname{Aut}\left(F_{m}\right)$ and $\varphi: F_{m} \rtimes_{\theta} \mathbb{Z}_{2} \rightarrow \operatorname{Aut}(\mathbb{Z})$ with $\left.\varphi\right|_{\mathbb{Z}_{2}}=\mathrm{id}_{\mathbb{Z}_{2}}$, we classify all realizable pairs $(\theta, \varphi)$, i.e., in view of Lemma 3.1, pairs $(\theta, \varphi)$ for which it does not exist $g \in F_{m}$ such that

$$
\left\{\begin{array}{l}
\theta\left(1_{2}\right)(g)=g^{-1} \\
\varphi(g, 0)=1_{2}
\end{array}\right.
$$

First, we recall a very useful result by Dyer and Scott [8, Theorem 3].

Theorem 3.3. Let $F$ be any free group, $\theta: \mathbb{Z}_{2} \rightarrow \operatorname{Aut}(F)$ a homomorphism and $F^{\theta\left(1_{2}\right)}<F$ the fixed point subgroup of the automorphism $\theta\left(1_{2}\right)$. Then there is a decomposition

$$
F=F^{\theta\left(1_{2}\right)} *\left(*_{i \in I} F_{i}\right) *\left(*_{\lambda \in \Lambda} F_{\lambda}\right)
$$

into the free product, where each factor is $\theta\left(1_{2}\right)$-invariant and:

(i) for each $i \in I, F_{i}=\left\langle x_{i, 1}, x_{i, 2}\right\rangle$ such that

$$
\theta\left(1_{2}\right)\left(x_{i, r}\right)=x_{i, r+1(\bmod 2)} \text { for } r=1,2
$$


(ii) for each $\lambda \in \Lambda$, there is a set $J_{\lambda}$ with $F_{\lambda}=\left\langle x_{\lambda}, y_{j} \mid j \in J_{\lambda}\right\rangle$ such that

$$
\begin{gathered}
\theta\left(1_{2}\right)\left(x_{\lambda}\right)=x_{\lambda}^{-1} \text { and } \\
\theta\left(1_{2}\right)\left(y_{j}\right)=x_{\lambda}^{-1} y_{j} x_{\lambda} \text { for } j \in J_{\lambda} \text { and } \lambda \in \Lambda .
\end{gathered}
$$

Basing on Lemma 3.1 and Theorem 3.3, we can provide a criterion to decide whether a pair is realizable or not. For this purpose the following is useful.

A well-known representation of $\operatorname{Aut}\left(F_{m}\right)$ is given by

$$
\rho_{m}: \operatorname{Aut}\left(F_{m}\right) \rightarrow \operatorname{Aut}\left(F_{m} / F_{m}^{\prime}\right) \cong G L_{m}(\mathbb{Z})
$$

where $F_{m}^{\prime}$ is the commutator subgroup of $F_{m}, G L_{m}(\mathbb{Z})$ the group of all invertible $m \times m$-matrices over $\mathbb{Z}$ and $\rho_{m}(\theta)$ is the automorphism of the free abelian group $F_{m} / F_{m}^{\prime} \cong \mathbb{Z}^{m}$ induced by $\theta \in \operatorname{Aut}\left(F_{m}\right)$. In view of [18], the group $\operatorname{Aut}\left(F_{m}\right)$ is finitely presented and $\rho_{m}$ is surjective. Because the inner automorphisms $\operatorname{Inn}\left(F_{m}\right) \subseteq \operatorname{ker} \rho_{m}$, there is the induced homomorphism $\tilde{\rho}_{m}: \operatorname{Out}\left(F_{m}\right)=\operatorname{Aut}\left(F_{m}\right) / \operatorname{Inn}\left(F_{m}\right) \rightarrow G L_{m}(\mathbb{Z})$ the kernel of which is called the classical Torelli group denoted by $\mathcal{T}_{m}$.

Write $I_{m}$ for the identity $m \times m$-matrix and define $m \times m$-matrices:

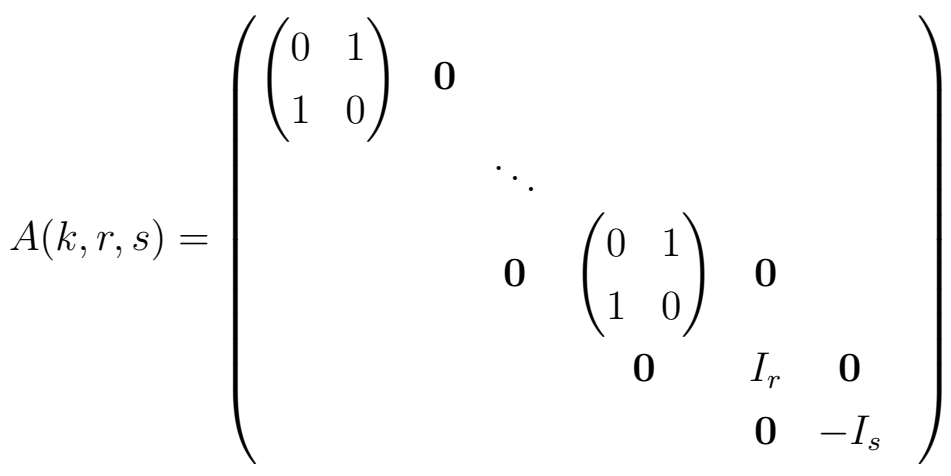

over integers which satisfy $A(k, r, s)^{2}=I_{m}$ with $k$ matrices $\left(\begin{array}{ll}0 & 1 \\ 1 & 0\end{array}\right)$ and $m=2 k+$ $r+s$.

Given $\theta: \mathbb{Z}_{2} \rightarrow \operatorname{Aut}\left(F_{m}\right)$ with $F_{m}=\left\langle x_{1}, \ldots, x_{m}\right\rangle$, by $\rho_{m}\left(\theta\left(1_{2}\right)\right)$ we intend to denote the matrix of the automorphism of the abelianization $F_{m}^{a b} \cong \mathbb{Z}^{m}$ with respect to the basis $\left\{\bar{x}_{1}, \ldots, \bar{x}_{m}\right\}$, where $\bar{x}_{i}$ is the projection of $x_{i}$ onto $\mathbb{Z}^{m}$ for $i=1, \ldots, m$. Because $\theta^{2}\left(1_{2}\right)=\operatorname{id}_{F_{m}}$ we have that $\rho_{m}\left(\theta\left(1_{2}\right)\right)^{2}=I_{m}$.

If $\theta: F_{m} \rightarrow F_{m}$ is given as follows: $\theta\left(x_{l}\right)=x_{l+1}$ and $\theta\left(x_{l+1}\right)=x_{l}$ for $l=$ $1,3, \ldots, 2 k-1 ; \theta\left(x_{l}\right)=x_{l}$ for $l=2 k+1,2 k+2, \ldots, 2 k+r$ and $\theta\left(x_{l}\right)=x_{l}^{-1}$ for $l=2 k+r+1,2 k+r+2, \ldots, 2 k+r+s$ then $\rho_{m}\left(\theta\left(1_{2}\right)\right)=A(k, r, s)$. Given $g \in F_{m}=\left\langle x_{1}, \ldots, x_{m}\right\rangle$, write $|g|_{x_{i}}$ for its $x_{i}$-exponent, i.e., $|g|_{x_{i}}=\sum_{k=1}^{t} r_{k}$ provided 
$x_{i}^{r_{k}}$ appears in $g$ for $i=1, \ldots, m$ and $k=1, \ldots, t$, and zero otherwise. Now, we are in position to state:

Theorem 3.4. Let $F_{m}=\left\langle x_{1}, \ldots, x_{m}\right\rangle$ be a free group with $m \geq 1, \theta: \mathbb{Z}_{2} \rightarrow \operatorname{Aut}\left(F_{m}\right)$ and $\varphi: F_{m} \rtimes_{\theta} \mathbb{Z}_{2} \rightarrow \operatorname{Aut}(\mathbb{Z})$ be homomorphisms such that $\rho_{m}\left(\theta\left(1_{2}\right)\right)=A(k, r, s)$ and $\left.\varphi\right|_{\mathbb{Z}_{2}}=\mathrm{id}_{\mathbb{Z}_{2}}$. Then the pair $(\theta, \varphi)$ is realizable if and only if $\varphi\left(x_{l}, 0\right)=0$ for $l=2 k+r+1, \ldots, 2 k+r+s$.

Proof. Consider the elements $g \in F_{m}$ such $\theta(g)=g^{-1}$. If $g$ belongs to the commutator subgroup of $F_{m}$ then $\varphi(g, 0)=0$. Because $\rho_{m}\left(\theta\left(1_{2}\right)\right)=A(k, r, s)$, the equation $\theta(g)=g^{-1}$ implies $A(k, r, s)(\bar{g})=-\bar{g}$, where $\bar{g}$ is the projection of $g$ into $\mathbb{Z}^{m}$. So we get:

$$
\begin{aligned}
& \left(\begin{array}{ccccccc}
\left(\begin{array}{ll}
0 & 1 \\
1 & 0
\end{array}\right) & \mathbf{0} & & & & & \\
& & & & & & \\
& & \mathbf{0} & \left(\begin{array}{cc}
0 & 1 \\
1 & 0
\end{array}\right) & \mathbf{0} & \\
& & & \mathbf{0} & I_{r} & \mathbf{0} \\
& & & & \mathbf{0} & -I_{s}
\end{array}\right)\left(\begin{array}{c}
|g|_{x_{1}} \\
\vdots \\
|g|_{x_{2 k}} \\
\vdots \\
|g|_{x_{2 k+r}} \\
\vdots \\
|g|_{x_{2 k+r+s}}
\end{array}\right)=\left(\begin{array}{c}
-|g|_{x_{1}} \\
\vdots \\
-|g|_{x_{2 k}} \\
\vdots \\
-|g|_{x_{2 k+r}} \\
\vdots \\
-|g|_{x_{2 k+r+s}}
\end{array}\right) . \\
& \text { Consequently, }\left\{\begin{array} { l } 
{ | g | _ { x _ { 1 } } = - | g | _ { x _ { 2 } } , } \\
{ \vdots } \\
{ | g | _ { x _ { 2 k - 1 } } = - g | _ { x _ { 2 k } } }
\end{array} \text { and } \left\{\begin{array}{l}
|g|_{x_{2 k+1}}=0 \\
\vdots \\
|g|_{x_{2 k+r}}=0 .
\end{array}\right.\right.
\end{aligned}
$$

Because $\varphi\left(x_{i}, 0\right)=\varphi\left(\theta\left(1_{2}\right)\left(x_{i}\right), 0\right)=\sum_{l=1}^{2 n+r}\left|\theta\left(1_{2}\right)\left(x_{i}\right)\right|_{x_{l}} \varphi\left(x_{l}, 0\right)$ for $i=1, \ldots, 2 k$, we derive that $\varphi\left(x_{i}, 0\right)= \begin{cases}\varphi\left(x_{i+1}, 0\right) & \text { if } i \text { is odd } \\ \varphi\left(x_{i-1}, 0\right) & \text { if } i \text { is even }\end{cases}$

Now we show one implication. Suppose that $\varphi\left(x_{l}, 0\right)=0$ for $l=2 k+r+1, \ldots, 2 k+$ $r+s$ and let us assume that $g \in F_{m}$ is a solution of the equation $\theta(g)=g^{-1}$. Then $\varphi(g, 0)=\sum_{i=1}^{2 k+r+s}|g|_{x_{i}} \varphi\left(x_{i}, 0\right)=\sum_{i=1}^{2 k}|g|_{x_{i}} \varphi\left(x_{i}, 0\right)=$ $|g|_{x_{1}} \varphi\left(x_{1}, 0\right)-|g|_{x_{1}} \varphi\left(x_{1}, 0\right)+\cdots+|g|_{x_{2 k-1}} \varphi\left(x_{2 k-1}, 0\right)-|g|_{x_{2 k-1}} \varphi\left(x_{2 k-1}, 0\right)=0$.

Therefore the system given by the Lemma 3.1 has no solution and the result follows.

To show the converse suppose the pair $(\theta, \varphi)$ is realizable. We know that $x_{l}$ for $l=2 k+r+1, \ldots, 2 k+r+s$ satisfies the equation $\theta(g)=g^{-1}$. Therefore by Lemma 3.1 it follows that $\varphi\left(x_{l}, 0\right)=0$ for $l=2 k+r+1, \ldots, 2 k+r+s$ and the proof is complete. 
Remark 3.5. (1) It is not difficult to show the Theorem 3.4 for $F \rtimes_{\theta} \mathbb{Z}_{2}$, where $F$ is a free group of arbitrary rank, once we adpte the hypothesis. Namely we assume that $\rho\left(\theta\left(1_{2}\right)\right)=A(k, r, s)$, where now we allow that $k, r, s$ can be infinite cardinals, and $\varphi\left(x_{l}, 0\right)=0$ for all indices $l$ which correspond to those, where the diagonal is -1 .

(2) Theorem 3.3 tells that there is at least one basis for $F_{m}$ such that the hypothesis $\rho_{m}\left(\theta\left(1_{2}\right)\right)=A(k, r, s)$ holds provided $m$ is finite. This is not clear if $F$ is of infinite rank. Although any free group $F$ and, by Corollary 3.2, the group $F \rtimes_{\theta} \mathbb{Z}_{2}$ act on a homotopy sphere $\Sigma(2 n)$.

4. Miscellanea. A very good survey about the subject below one can find in [11], where several questions are posed and discussed. In this section, we use some information from [11] and make related comments having in mind mainly actions on homotopy spheres $\Sigma(2 n)$.

We begin by recalling that by [16, Corollary 5.6], the Thompson group

$$
F=\left\langle x_{0}, x_{1}, \ldots \mid x_{i} x_{j} x_{i}^{-1}=x_{j+1}, i<j\right\rangle
$$

with $\operatorname{cd} F=\operatorname{vcd} F=\infty$ does not act freely and properly discontinuously on any $\mathbb{R}^{m} \times \mathbb{S}^{n}$. In fact, it is also true that $F$ does not act freely and properly discontinuously on any homotopy sphere $\Sigma(n)$. To see this, suppose that $F$ acts on some $\Sigma(n)$. Using the fact that for every positive integer $m$ the group $F$ contains a copy of the free abelian group $\mathbb{Z}^{m}$, Proposition 1.3 implies the inequality $\operatorname{dim} \Sigma(n) \geq \operatorname{vcd} \mathbb{Z}^{m}+n=$ $n+m$. But this is not possible for an arbitrary $m$ and the result follows.

In view of [5], this countable group $F$ has periodic cohomology in the sense that $H^{i}(F, \mathbb{Z}) \cong H^{i+2}(F, \mathbb{Z})$ for all $i>1$. But we are unable to show its periodicity after some steps (in the sense of [28]). Using [5], it can be shown, without too much difficulties that this isomorphism cannot be realized by means of the cup product.

S. Prassidis has shown in the paragraph following [20, Theorem 10] that:

Theorem 4.1. There exist discrete groups $G$ with $\operatorname{vcd} G=\infty$ which act freely and properly on some $\mathbb{R}^{m} \times \mathbb{S}^{n}$.

The action given in [20] is free, properly discontinuous but not co-compact. Then F.T. Farrell and C.W. Stark [9, Theorem 1] showed: 
Theorem 4.2. For each $m \geq 2$ and $n \geq m(m+1)$, there are smooth closed manifolds with universal covering spaces $\mathbb{R}^{m} \times \mathbb{S}^{2 n-1}$ and fundamental group of infinite virtual cohomological dimension.

Groups from the results above are torsion with vcd $G=\infty$ and in view of Proposition 1.2 they cannot act on any $\Sigma(2 n)$, in particular on any $\mathbb{R}^{m} \times \mathbb{S}^{2 n}$. But it is natural to ask: can a torsion-free group $G$ with $\operatorname{cd} G=\infty$ acts (possibly co-compactly), freely and properly discontinuously on some $\mathbb{R}^{m} \times \mathbb{S}^{n}$ ? By private communication with F.X. Connolly and S. Prasidis this question is unsettled.

Several of the questions and results above, can be studied if we restrict to the family of homotopy spheres $\Sigma(2 n)$. Taking into account [14, Theorem 5.2], we close this paper with:

Question 4.3. Suppose that a group $G$ acts, freely and properly discontinuously (possibly co-compactly) on some $\Sigma(2 n)$ with $\operatorname{dim} \Sigma(2 n) \leq m+2 n$. Does it follow that $\operatorname{vcd} G \leq m$ ?

Certainly, the proof of [14, Theorem 5.2 (1)] leads to vcd $G \leq m$ provided vcd $G<$ $\infty$. Notice that Proposition 2.3 yields vcd $G \leq 1$ for any virtually cyclic group $G$ and $\operatorname{dim} \Sigma(2 n)<\infty$. Further, by the proof of [14, Corollary 7.2] the answer to Question 4.3 is affirmative for $m=0,1$.

\section{REFERENCES}

[1] A. Adem, R.J. Milgam, Cohomology of Finite Groups, Springer-Verlag, New York-HeidelbergBerlin (1994).

[2] A. Adem, J.H. Smith, Periodic complexes and group actions, Ann. of Math. 154 (2001), 407435.

[3] J. Bjørn, S. Kwasik, Free involutions on $\mathbb{S}^{1} \times \mathbb{S}^{n}$, Math. Ann. 351, No. 2 (2011), 281-303.

[4] K.S. Brown, Cohomology of groups, Springer-Verlag, New York-Heidelberg-Berlin, 1982.

[5] - The homology of Richard Thompson's group F. Topological and asymptotic aspects of group theory, 47-59, Contemp. Math., 394, Amer. Math. Soc., Providence, RI, 2006.

[6] F.X. Connolly, S. Prassidis, Groups which act freely on $\mathbb{R}^{m} \times \mathbb{S}^{n-1}$. Topology 28, no. 2 (1989), 133-148.

[7] M. Culler, Finite groups of outer automorphisms of a free group, Contributions to group theory, 197-207, Contemp. Math. 33, Amer. Math. Soc., Providence, RI, 1984.

[8] J.L. Dyer, G.P. Scott, Periodic automorphisms of free groups, Comm. Algebra 3(3) (1975), 195-201.

[9] F.T. Farrell, C.W. Stark, Cocompact spherical Euclidean spaceform grops of infinite VCD, Bull. London Math. Soc. 25 (1993), 189-192. 
[10] M. Golasiński, D.L. Gonçalves, R. Jiménez, Free and properly discontinuous actions of discrete groups on homotopy circles (submitted).

[11] I. Hambleton, E.K. Pedersen, More examples of discrete co-compact group action, arXiv:1301.7267v1 [math.GT].

[12] D.L. Johnson, Presentations of groups, Second edition. London Mathematical Society Student Texts, 15. Cambridge University Press, Cambridge, 1997.

[13] F.E.A. Johnson, Manifolds of homotopy type $K(\pi, 1)$. I, Proc. Cambridge Philos. Soc. 70 (1971), 387-393.

[14] J.B. Lee, Transformtion groups on $\mathbb{S}^{n} \times \mathbb{R}^{m}$, Topology Appl. 53 (1993), 187-204.

[15] J. Milnor, Groups which act on $\mathbb{S}^{n}$ without fixed points, Amer. J. Math. 79 (1957), 623-630.

[16] G. Mislin and O. Talelli, On groups which act freely and properly on finite dimensional homotopy spheres, Computational and geometric aspects of modern algebra (Edinburgh, 1998), 208228, London Math. Soc. Lecture Note Ser., 275, Cambridge Univ. Press, Cambridge (2000).

[17] J. Nielsen, Die Isomorphismen der allgemeinen Gruppe mit zwei Erzeugenden, Math. Ann. 78, (1917), 385-397.

[18] — Die Isomorphismengruppe der freien Gruppen, Math. Ann. 91 (1924), 169-209.

[19] - Die Gruppe der dreidimensionalen Gittertransformationen, Kgl. Danske Videnskabernes Selskab., Math. Fys. Meddelelser V, 12 (1924), 1-29.

[20] S. Prassidis, Groups with infinite virtual cohomological dimension which act freely on $\mathbb{R}^{m} \times$ $\mathbb{S}^{n-1}$, J. Pure Appl. Algebra 78, no. 1 (1992), 85-100.

[21] C.A. Robinson, Moore-Postnikov systems for non-simple fibrations, Illinois J. Math. 16 (1972), 234-242.

[22] C. Scott, C.T.C. Wall, Topological methods in group theory. Homological group theory (Proc. Sympos., Durham, 1977), pp. 137-203, London Math. Soc. Lecture Note Ser., 36, Cambridge Univ. Press, Cambridge-New York, 1979.

[23] P.-J. Serre, Cohomologie des groupes discrets, Ann. of Math. Studies 70 (1971), 77-169.

[24] J. Stallings, On torsion-free groups with infinitely many ends, Ann. of Math. 88 (1968), 312334.

[25] R.G. Swan, Periodic resolutions for finite groups, Ann. of Math. 72 (1960), 261-291.

[26] — A new method in fixed point theory, Comment. Math. Helv. 34 (1960), 1-16.

[27] — Groups of cohomological dimension one, J. Algebra 12 (1969), 585-601.

[28] O. Talleli, On cohomological periodicity for infinite groups, Comment. Math. Helv. 55 (1980), 178-192.

[29] - , Periodicity in cohomology and free and proper actions on $\mathbb{R}^{n} \times \mathbb{S}^{m}$, Campbell, C.M. (ed.) et al., Groups St. Andrews 1997 in Bath. Selected papers of the international conference, Bath, UK, July 26-August 09, 1997, vol. 2. Cambridge: Cambridge University Press. Lond. Math. Soc. Lect. Note Ser. 261 (1999), 701-717.

[30] J.L. Tollefson, The compact 3-manifolds covered by $\mathbb{S}^{2} \times \mathbb{R}^{1}$, Proc. Amer. Math. Soc. 45 (1974), 461-462.

[31] C.T.C. Wall, Poincaré complexes I, Ann. Math. 86 (1967), 213-245.

[32] — , Periodic projective resolutions, Proc. London Math. Soc. (3) 39 (1979), 509-553. 
[33] B. Zimmermann, Über Homöomorphism n-dimensionaler Henkelkörper und endliche Erweiterungen von Schottky-Gruppen, Comm. Helv. 56 (1981), 474-486.

Institute of Mathematics

Casimir the Great University

pl. Weyssenhoffa 11

85-072 Bydgoszcz, Poland

e-mail: marek@ukw.edu.pl

Department of Mathematics-IME

University of São Paulo

Caixa Postal 66.281-AG. Cidade de São Paulo

05314-970 São Paulo, Brasil

e-mail: dlgoncal@ime.usp.br

Instituto de Matemáticas, Unidad Oaxaca

Universidad Nacional Autónoma de México

Oaxaca, Oax. México

e-mail: rolando@matcuer.unam.mx 